\title{
New bilayer-coated microbead system for controlled release of 5-aminosalicylic acid
}

\author{
Anna Karewicz • Joanna Lęowik • \\ Maria Nowakowska
}

Received: 22 March 2010/Revised: 6 August 2010/Accepted: 18 August 2010/

Published online: 27 August 2010

(C) The Author(s) 2010. This article is published with open access at Springerlink.com

\begin{abstract}
A new bilayer-coated microbeads for oral delivery of 5-aminosalicylic acid (5-ASA) were prepared from naturally occurring polysaccharides via emulsification method. 5-ASA was successfully encapsulated in chitosan- $\lambda$-carrageenancoated alginate microbeads. The morphology and sizes of microparticles were studied using optical microscopy and dynamic light scattering. Drug release profiles were followed using high-performance liquid chromatography (HPLC) with spectrophotometric detection. The effect of chitosan thin layer and chitosan- $\lambda$-carrageenan bilayer on the drug release was studied at $\mathrm{pH}$ values simulating these characteristic for stomach $(\mathrm{pH}=1.2)$ and intestine $(\mathrm{pH}=7.4)$. Chitosan layer slightly increased 5-ASA release at $\mathrm{pH}=1.2$, whereas double-layer external coating decreased the release rates. Due to the bilayer properties, the micro-carriers were additionally protected against acidic conditions of the stomach and drug release in the intestine could be better controlled.
\end{abstract}

Keywords Alginate $\cdot$ Chitosan $\cdot \lambda$-carrageenan $\cdot$ Microparticles $\cdot$ Drug delivery Ultrathin layers

\section{Introduction}

5-Aminosalicylic acid (5-ASA) has been successfully used for over a decade in the treatment of inflammatory bowel diseases (IBD) such as ulcerative colitis or Crohn's disease. Patients suffering from these maladies are exposed to increased risk for the development of colorectal cancer (CRC) and the risk becomes higher with the extent and duration of the disease. Experimental and epidemiological

\footnotetext{
A. Karewicz $(\bowtie) \cdot$ J. Łęgowik $\cdot$ M. Nowakowska

Nanotechnology of Polymers and Biomaterials Group, Physical Chemistry Department, Faculty of Chemistry, Jagiellonian University, Ingardena 3, 30-060 Cracow, Poland e-mail: karewicz@chemia.uj.edu.pl
} 
studies suggest that long-term regular intake of 5-ASA may reduce the occurrence of colorectal cancer in patients with IBD. Bernstein et al. [1] have studied the effect of various chemical agents on CRC chemoprevention and demonstrated the strong protective association of regular 5-ASA therapy reducing cancer risk by $81 \%$. These findings were followed by intense studies on mechanism of action explaining therapeutic effect of 5-ASA on CRC prevention. Several articles have been published showing that 5-ASA reduces significantly the growth and survival of CRC cells [2-4].

An important factor in the successful prevention of colorectal cancer is patient's compliance. Strong adverse effects (hepatitis, blood dyscrasias, pancreatitis, or interstitial nephritis) associated with oral 5-ASA therapy [5] as well as a need of regular long-term treatment make patient's compliance difficult to achieve. Decreasing the 5-ASA dose and frequency of intake and developing the optimal matrix for decreasing adverse effects would, therefore, be of great importance. When administered orally, 5-aminosalicylic acid should be delivered in the form allowing reduction of its systemic absorption and degradation while drug is passing the stomach. 5-ASA is known to be rapidly absorbed from small intestine, so a sufficient protection in this part of the gastrointestinal tract is also necessary to assure optimal delivery of the active moiety to the colon. Lakatos et al. [6] have reported that one-daily formulation of 5-ASA, delayed-release tablets of mesalazine utilizing Multi-Matrix System (MMX) technology, has been recently approved in the US for the induction and maintenance of remission in patients with active ulcerative colitis. However, the total systemic absorption of the new drug does not show any supremacy over the other 5-ASA formulations.

Naturally occurring polysaccharides, such as alginate, $\lambda$-carrageenan, or chitosan, are widely used in drug delivery due to their biocompatibility, mucoadhesive properties, abundance in nature and low cost. Sodium alginate is well known for its ability to form $\mathrm{pH}$-sensitive hydrogels under mild conditions upon addition of calcium ions. Its swelling in acidic conditions typical for stomach $(\mathrm{pH}=1.2)$ is minimal, assuring good protection of the compound embedded in the gel structure. However, considering the practical applications, one should ensure that the extent of swelling can be also controlled at higher $\mathrm{pH}$, characteristic for the small intestine and colon, where alginate gels swell considerably. We suggest that this can be achieved by coating of alginate matrix with chitosan, natural polymer whose solubility at $\mathrm{pH}$ higher than 6.5 is considerably limited. The presence of chitosan should suppress the microsphere erosion in small intestine and colon leading to significant decrease and better control over 5-aminosalicylic acid release.

Both polymers, as well as their combination, have been already applied to obtain microbeads [7]. Also, multilayer systems composed of both polysaccharides were applied successfully to deliver drugs (e.g. ampicillin) [8]. There are, however, very few articles on controlled delivery of 5-ASA using microspherical systems. Moreover, in those systems the commercially available enteric coating materials (such as Eudragit or Aquacoat) are applied as single outer layer to protect the drug while passing of the stomach [9].

Here we propose alginate-chitosan- $\lambda$-carrageenan microparticulate system for controlled delivery of 5-ASA based on ultrathin double-layered external coating of 
the alginate matrix. While the internal chitosan layer should decrease drug's release in jejunum and the ileum of the gut, the main role of the outer layer would be to protect the internal layer from the dissolution in the stomach. Our choice was to use $\lambda$-carrageenan. Carrageenans are polysaccharides extracted from red seaweeds and widely used in food and pharmaceutical industry. There are three main classes of carrageenans: kappa, iota, and lambda, differing in the number and position of the ester sulfate groups on the repeating galactose units. In our studies $\lambda$-carrageenan was chosen due to the highest level of ester sulfate. This property provides good water solubility and high charge density of the polymer chain. In our procedure, first the Ca-alginate microbeads containing 5-ASA were produced and then they were coated with the external thin layers (chitosan followed by $\lambda$-carrageenan). This allowed for studying the effect of the inner and outer layer on the release profiles in $\mathrm{pH}$ simulating body fluids (stomach and intestine). Considering the fact that the relative amounts of chitosan and $\lambda$-carrageenan present in the microparticles are extremely low, the bulk properties of the alginate gel matrix are not modified.

\section{Experimental}

\section{Materials}

5-Aminosalicylic acid (Sigma, purity $\sim 99 \%$ ), alginic acid sodium salt from brown algae (sodium alginate, Sigma, viscosity of the $2 \%$ solution at $25{ }^{\circ} \mathrm{C}: \sim 2,000 \mathrm{cP}$ ), chitosan (Aldrich, low molecular weight, 75-85\% deacetylated, viscosity of $1 \%$ solution in $1 \%$ acetic acid at at $25^{\circ} \mathrm{C}: 20-200 \mathrm{cP}$, ), $\lambda$-carrageenan (BioChemika (Sigma)), calcium chloride (Sigma-Aldrich, min. 93\%, granular, anhydrous), methanol for chromatographic measurements (Fluka, for HPLC, gradient grade, $\geq 99.8 \%$ (GC)), ethylenediaminetetraacetic acid tetrasodium salt hydrate (EDTA, SigmaUltra, $\geq 99 \%(\mathrm{KT})$ ), distilled water was of MilliQ grade.

\section{Preparation of uncoated microbeads}

5-ASA-loaded alginate microparticles were prepared by emulsification method using calcium chloride as a crosslinking agent. $2.808 \mathrm{~g}$ of stabilizing agent (Tween 85) was added to $160 \mathrm{ml}$ of cyclohexane and stirred until completely dissolved. Then, aqueous solution containing $0.4 \mathrm{~g}(1.25 \mathrm{wt} \%)$ of sodium alginate and $100 \mathrm{mg}$ (2.2 $\mathrm{mM})$ of 5-ASA was added dropwise. The mixture was then stirred at $800 \mathrm{rpm}$ for about $10 \mathrm{~min}$ to obtain stable emulsion. Next, $30 \mathrm{ml}$ of $0.2 \mathrm{M}$ aqueous solution of calcium chloride was added dropwise to the reaction mixture to obtain crosslinked alginate microparticles encapsulating 5-ASA molecules in the calcium alginate matrix. Emulsion was then stirred for another $30 \mathrm{~min}$. After this time, stirring was switched off and emulsion was left for $1 \mathrm{~h}$ to allow microspheres to harden. The microparticles thus formed were then filtered and washed with distilled water and 2-propanol. 
Coating procedure

5-ASA-loaded microbeads were coated with thin layers of chitosan and $\lambda$-carrageenan. For this purpose, the solutions of chitosan and $\lambda$-carrageenan were prepared in $0.02 \mathrm{M}$ acetic acid with addition of $\mathrm{NaCl}(0.15 \mathrm{M})$ to assure invariability of the ionic strength. $\mathrm{pH}$ of both solutions was adjusted to 5.0 and then they were filtered up by using syringe filter (pore size: $0.45 \mu \mathrm{m}$ ). To obtain each layer, the microspheres were introduced to the solution of the polyelectrolyte and mixed for about $30 \mathrm{~min}$. Then, they were filtered up and washed with distilled water.

Optical microscopy measurements

Optical microscope imaging was performed using Nikon Eclipse TE2000 Inverted Research Microscope System. All the images were obtained in the air using Plan Achromat 50x objective. A small amount of the sample studied (ca $2 \mathrm{mg}$ ) was dispersed in water using ultrasonic bath and spread over the glass slide directly before the measurement. The diameter of the microbeads was measured as a mean value for 100 microbeads.

\section{SEM measurements}

Field-emission scanning electron microscopy (FE-SEM, Hitachi S-4700) equipped with energy dispersive X-ray spectroscope (EDS) was used to study the morphology and microstructure of microbeads (magnifications from $5,000 \times$ to $20,000 \times$ ). Sample was dispersed and diluted in water, spread over the thin glass slide and dried in the stream of gaseous nitrogen directly prior to measurement.

Encapsulation efficiency measurements

Encapsulation efficiency was measured by dissolving the precisely weighted sample of the microspheres in $5 \mathrm{mM}$ EDTA solution containing $\mathrm{NaCl}(100 \mathrm{mM})$ at $\mathrm{pH}=7.4$. The sample was then stirred at room temperature until the microparticles were completely dissolved. $1 \mathrm{ml}$ of the resulting solution was then tested using HPLC method in order to measure the concentration of 5-ASA. Before each measurement a calibration curve was obtained for 10 standard solutions of 5-aminosalicylic acid. Encapsulation efficiency (EE) was calculated from the following equation:

$$
\mathrm{EE}=\left(W_{\text {enc }} / W_{\text {int }}\right) \times 100 \%
$$

where $W_{\text {enc }}$ amount of 5-ASA encapsulated in macrospheres, $W_{\text {int }}$ amount of 5-ASA in the reaction mixture

DLS measurements

The light scattering measurements were performed using Malvern Instruments ZETASIZER NANO-ZS. The samples were prepared in aqueous solution, filtered 
through Chromafil filter $(0.45 \mu \mathrm{m})$ and measured at room temperature. Data were analyzed using the Malvern software and were the average from six measurements. All the experiments were performed in triplicate.

\section{Release studies}

To study the release of 5-ASA from the microspheres, the $15 \mathrm{mg}$ samples were placed in $7 \mathrm{ml}$ plastic centrifuge tubes and $3 \mathrm{ml}$ of the medium solution was added. A $0.1 \mathrm{M}$ solution of $\mathrm{HCl}$ was used to simulate the $\mathrm{pH}$ of the stomach while PBS solution $(\mathrm{pH}=6.8)$ was used to simulate the intestine $\mathrm{pH}$. Each sample was then left in the water bath at $37{ }^{\circ} \mathrm{C}$, while constantly stirred. After defined period of time, the tube with the sample was centrifuged and all the solution withdrawn. The solution was then analyzed to determine the drug concentration. Then, a new portion of the release medium $(3 \mathrm{~mL})$ was added to the microsphere sample and the release studies were continued.

Drug concentration measurements were done using HPLC method. The analysis was performed using C18 RP column and UV/Vis spectrophotometric detection at $317 \mathrm{~nm}$. Water/citric buffer $(10 \mathrm{mM} ; \mathrm{pH}=2.75) /$ acetonitrile/methanol were mixed in proportion: 13:1:3:3 (v/v/v/v) and the obtained solution was used as a mobile phase. The flow rate was $0.5 \mathrm{~mL} / \mathrm{min}$. All the samples were measured in triplicate.

\section{Results}

The microbeads prepared using emulsification method were characterized using the optical microscopy to determine their morphology and size distribution. As can be seen at the images presented in Fig. 1a and b, the spherical objects of micrometric size $(2-10 \mu \mathrm{m})$ were obtained, although in some cases small morphological irregularities could be also observed. This can be explained considering soft and rather fragile nature of the hydrogel structure of Ca-alginate particles The microspheres show a moderate tendency for aggregation. Incorporation of 5-ASA into microspheres influences their morphology significantly (Fig. 1c, d). 5-ASA encapsulating microparticles show well-defined spherical shape, but they have higher size dispersion. The average bead diameter was estimated to be $9.2 \mu \mathrm{m}$. 5-ASA encapsulation efficiency was $20 \%$.

Those observations were confirmed by using Scanning Electron Microscopy. SEM images (Fig. 2a-c) show symmetrical spherical objects of the smooth surface and the size of ca $2-3 \mu \mathrm{m}$. To obtain such images the particles had to be sufficiently hydrated. Upon high pressures in the SEM apparatus fast dehydration occurred, followed by the microsphere subsidence due to the collapse of the hydrogel structure (Fig. 2d).

To increase the control over the drug release from the particles, two additional thin polysaccharide layers were introduced; first a chitosan and then $\lambda$-carrageenan layer. For this purpose, the electrostatic self assembly technique, known also as a layer-by-layer method $[10,11]$, was used. To verify the presence of the coating, zeta 
a
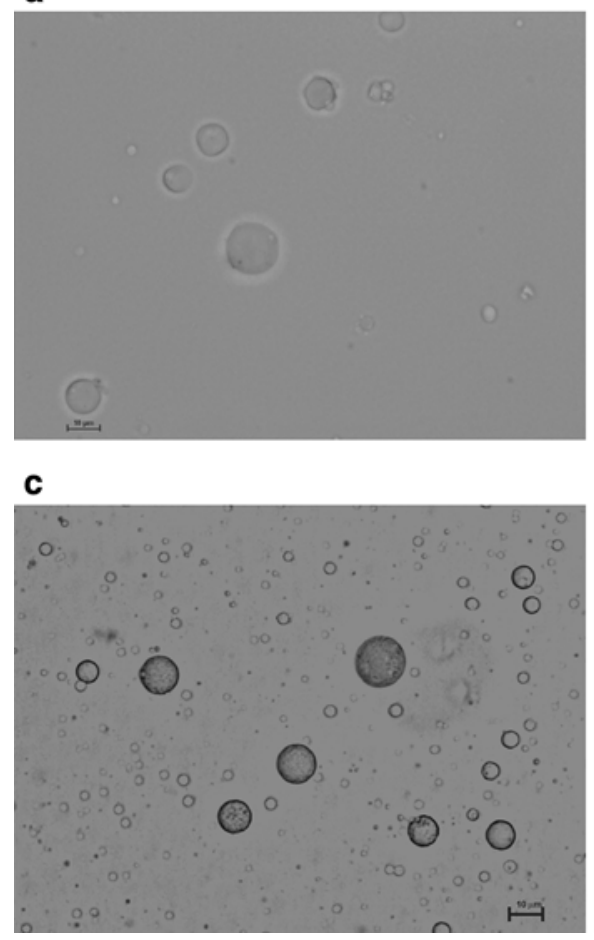

b

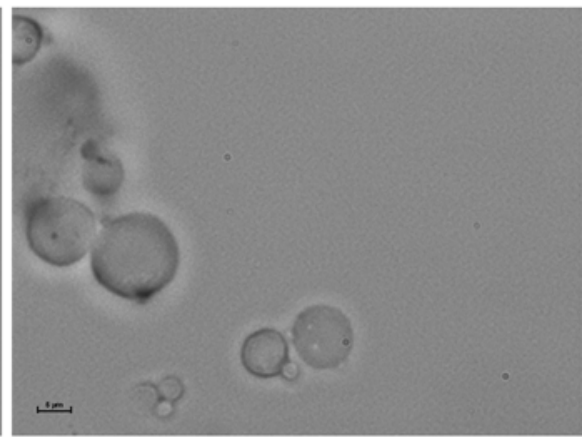

d

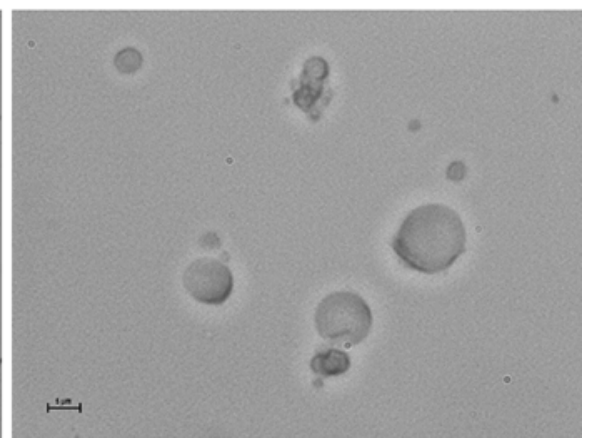

Fig. 1 Optical microscope images of uncoated alginate microparticles obtained for: a non-loaded particles $(\times 50$ magnification $)$, b non-loaded particles $(\times 100)$, c 5-ASA-loaded particles $(\times 50)$, and $\mathbf{d}$ of alginate microparticles obtained for chitosan/carragenan bilayer-coated, 5-ASA-loaded particles $(\times 100)$

potential measurements were performed, after the microparticles were coated with each layer (chitosan and then $\lambda$-carrageenan; see Table 1 ). As expected, in the neutral $\mathrm{pH}$ uncoated microbeads show negative surface charge due to the presence of ionized carboxylic groups of the alginate matrix, whereas in the acidic media the ionization is suppressed and zeta potential values are close to zero. In acidic chitosan solution the polymeric chains are positively charged and zeta potential of chitosan-coated microspheres is low, but positive. At $\mathrm{pH}=6.8$, zeta potential of chitosan-coated microparticles is still negative, but its absolute value is lower than that for uncoated microparticles.

Introduction of the outer carrageenan layer decreases zeta potential. Carrageenan sulfonate groups are ionized regardless of the $\mathrm{pH}$. The observed difference results from the compensation of alginate-chitosan charges. The fact that both layers have limited effect on the zeta potential of the microspheres can be explained by the fact that both of them are very thin and taking into account the fact that polymeric chains of the polysaccharides used to form the layer can easily interpenetrate the outer part of the microbead.

Release profiles were obtained for two different $\mathrm{pH}$ values: 1.2 and 6.8, typical for stomach and intestine environment, respectively. 5-ASA concentration was 
a

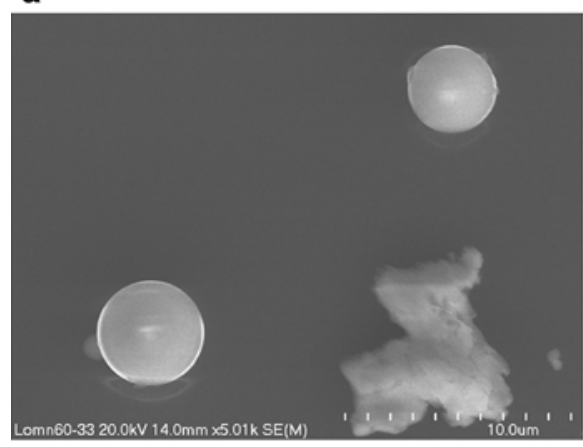

C

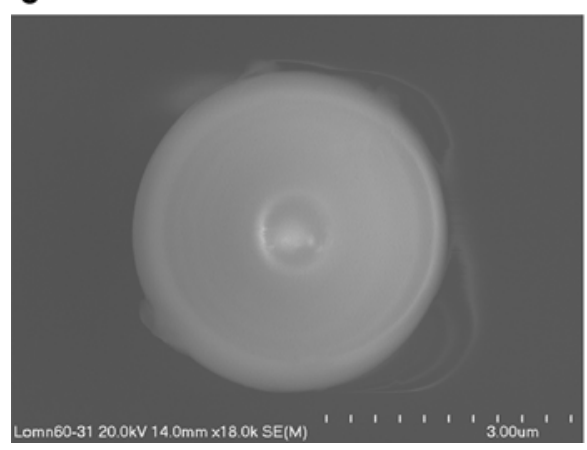

b

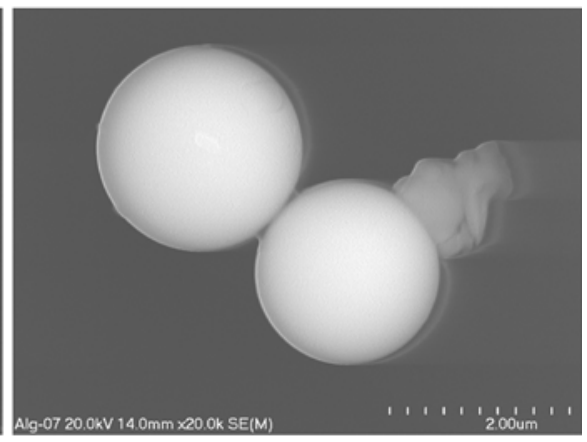

d

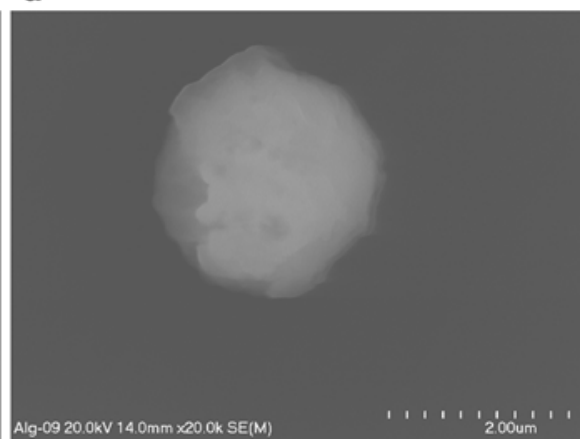

Fig. 2 SEM images of 5-ASA-loaded, uncoated alginate microparticles: a $\times 5,000$ magnification-two non-aggregated wet microparticles; $\mathbf{b} \times 20,000$ magnification-two aggregated wet particles of spherical shape and smooth surface; $\mathbf{c} \times 18,000$ magnification-single wet particle of highly spherical shape (reflections due to the wetness), $\mathbf{d} \times 20,000$ magnification-single dry particle with uneven surface and irregular shape due to the water loss of the hydrogel matrix

Table 1 Zeta potential $(\zeta)$ for uncoated and coated alginate microbeads

\begin{tabular}{lrr}
\hline Coating type & $\begin{array}{l}\text { Zeta potential } \\
\text { at } \mathrm{pH}=1.2\end{array}$ & $\begin{array}{l}\text { Zeta potential } \\
\text { at } \mathrm{pH}=6.8\end{array}$ \\
\hline None & $-4.5 \pm 1.7$ & $-34.2 \pm 1.7$ \\
Chitosan & $0.5 \pm 2.2$ & $-29.7 \pm 2.1$ \\
Chitosan and carrageenan & $-1.1 \pm 1.6$ & $-30.5 \pm 1.2$ \\
\hline
\end{tabular}

measured using HPLC method. Calibration curve for ten standards was done prior to each measurement series and each time excellent fit was obtained $\left(R^{2} \geq 0.99\right)$.

The release of 5-ASA from the obtained microspheres is very effective in the first $2 \mathrm{~h}$. Only $10 \%$ or less is retained after this time and then the release slows down significantly. As can be seen in Fig. 3b, in the stomach-like environment $(\mathrm{pH}=1.2)$ in the first $2 \mathrm{~h}$ chitosan coating has only slight accelerating effect on the release of 5-ASA in comparison to the release from the uncoated microbeads. For the longer release times, the profiles (Fig. 3a) are identical for both the types of microparticles 

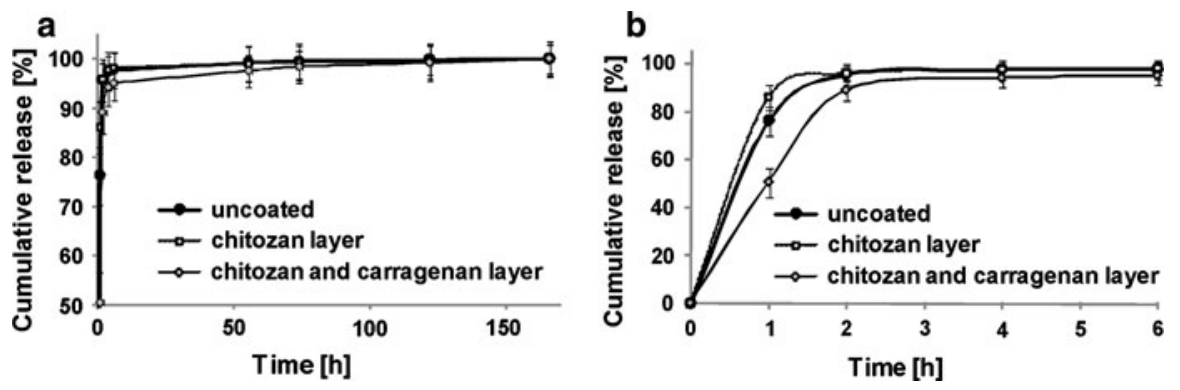

Fig. 3 Release profiles for uncoated and coated microspheres at $\mathrm{pH}=1.2$; $\mathbf{a}$ full profiles, $\mathbf{b}$ profiles for the first $6 \mathrm{~h}$; uncoated (filled circle), chitosan-coated (open square) and chitosan- $\lambda$-carrageenan-coated (open diamond) microbeads

suggesting complete dissolution of the outer layer. Introduction of the chitosancarrageenan bilayer, however, slows down the release process considerably (by ca $40 \%$ in the first $2 \mathrm{~h}$ ). Slow release can be observed even after 2 days, confirming that the bilayer is relatively stable in acidic media. After 3 days practically all 5-ASA is released, most probably due to the matrix degradation process.

Figure 4 shows the release profile at the $\mathrm{pH}=6.8$, simulating that of the human intestine. The presence of chitosan layer accelerates slightly the drug release in the first $2 \mathrm{~h}$. The chitosan-carrageenan double-layer coating, however, significantly slows down the drug release under the intestinal-like $\mathrm{pH}(\mathrm{pH}=6.8)$. 5-ASA is still released from those microspheres even after 2 days.

Figure 5 shows the differences in the release profiles of 5-ASA from the bilayercoated microparticles in various $\mathrm{pH}$. For the first $90 \mathrm{~min}$ the release is slower in the acidic medium and then the tendency is reversed. After $100 \mathrm{~h}$ the profiles intercross again but at this stage almost all the drug is already released in both media.

\section{Discussion}

As one can expect, the additional thin layers of chitosan and carrageenan deposited on microbeads do not modify their morphology, as observed using optical microscopy. However, the substantial differences in the drug release profiles from the uncoated and coated particles are observed, suggesting that the significant modification of the microparticles' surface has occurred.

Increase in the release rate of 5-ASA from chitosan-coated beads in $\mathrm{pH}=1.2$ may be explained taking into account the possibility of partial dissolution of the chitosan layer in acidic media, inducing some morphological changes in the alginate matrix. The similar release profiles for chitosan-coated and uncoated microspheres at that $\mathrm{pH}$ can be caused by the complete dissolution of the outer layer. In the environment of intestine the chitosan layer is more compact and cannot be easily penetrated by water. As a result, it slows down the drug release. After $2 \mathrm{~h}$ the release profiles for uncoated and chitosan-coated beads become almost identical. 

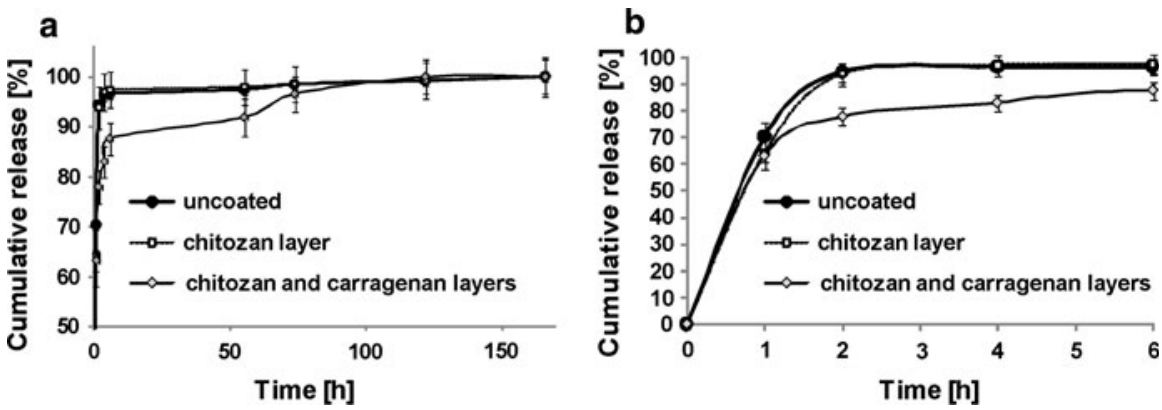

Fig. 4 Release profiles for uncoated and coated microspheres at $\mathrm{pH}=6.8$; $\mathbf{a}$ full profiles, $\mathbf{b}$ profiles for the first $6 \mathrm{~h}$; uncoated (filled circle), chitosan-coated (open square) and chitosan- $\lambda$-carrageenan-coated (open diamond) microbeads
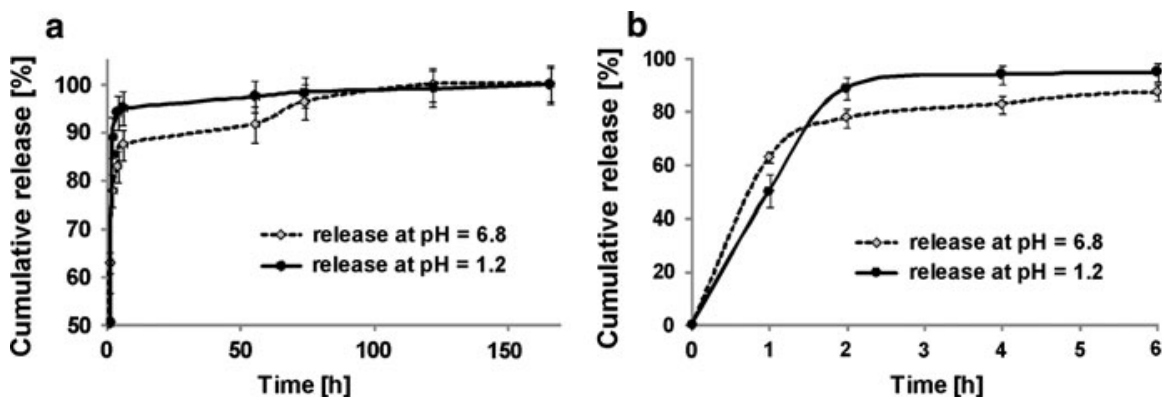

Fig. 5 Release profiles of bilayer-coated microbeads in $\mathrm{pH}$ characteristic for stomach (filled circle) and intestine (open diamond); a full profiles, b profiles for the first $6 \mathrm{~h}$

Again that may be explained considering the interpenetration of the chitosan chain into alginate matrix and possibility of slow degradation of the thin chitosan layer with the time.

Upon addition of the second layer, the release rate decreases in both environments. The profiles for double-layer-coated beads show that at the beginning more drug is released at $\mathrm{pH}=6.8$ and after 90 min the inversion of the tendency is observed. Since it has been estimated that it takes ca $2 \mathrm{~h}$ for the 5-ASA carriers to pass the stomach after their administration, this tendency appears to be advantageous.

To characterize the release mechanisms, the Higuchi [12] and Peppas [13] kinetic models are widely used. Both models, however, are short time approximations and their use is limited to the first $60 \%$ of the release. The Weibull model, on the other hand, is an alternative empirical equation that can be used to describe the entire set of experimental data. Although its parameters do not have direct physical interpretation, Papadopoulou and coworkers [14] in their work provided a link between the values of $b$ and the mechanism of the release. The Weibull model fits to the experimental profiles obtained for uncoated and coated microspheres are presented in Table 2. 
Table 2 Parameters and coefficients obtained for Weibull release kinetic model fitted to the experimental 5-ASA release profiles at $\mathrm{pH}$ characteristic for stomach $(\mathrm{pH}=1.2)$ and intestine $(\mathrm{pH}=6.8)$

Weibull kinetic model equation: $\frac{M_{\mathrm{t}}}{M_{\infty}}=a\left(1-\exp \left(-(k t)^{b}\right)\right)$

\begin{tabular}{llll}
\hline & $\begin{array}{l}\text { Uncoated } \\
\text { particles }\end{array}$ & $\begin{array}{l}\text { Chitosan-coated } \\
\text { particles }\end{array}$ & $\begin{array}{l}\text { Chitosan/ } \\
\text { carrageenan-coated } \\
\text { particles }\end{array}$ \\
\hline $\mathrm{pH}=1.2$ & & \\
$a$ & $99.0 \pm 0.44$ & $99.1 \pm 0.32$ & $97.4 \pm 0.96$ \\
$b$ & $1.2 \pm 0.15$ & $0.70 \pm 0.097$ & $1.7 \pm 0.20$ \\
$K$ & $1.38 \pm 0.074$ & $2.8 \pm 0.43$ & $0.84 \pm 0.030$ \\
$R^{2}$ & 0.9872 & 0.9816 & 0.9858 \\
$\mathrm{pH}=6.8$ & & \\
$a$ & $97.9 \pm 0.61$ & $98.4 \pm 0.47$ & $970 \pm 1.8$ \\
$b$ & $1.4 \pm 0.20$ & $1.5 \pm 0.13$ & $0.42 \pm 0.089$ \\
$K$ & $1.19 \pm 0.053$ & $1.03 \pm 0.023$ & $1.3 \pm 0.30$ \\
$R^{2}$ & 0.9834 & 0.9936 & 0.9930 \\
\hline
\end{tabular}

For the majority of the samples, the calculated $b$ values were above 1 . This is indicative of complex release mechanism. There are, however, two exceptions. The release from the chitosan-coated particles in acidic medium can be associated with drug diffusion in normal Euclidian space $(b=0.7)$ [15]. The lowest $b$ value was obtained for the bilayer-coated microspheres in $\mathrm{pH}=6.8(b=0.42)$ and according to Kosmidis et al. [15] in this case the release can be described by the diffusion mechanism in fractal or disordered substrate.

\section{Conclusions}

We have obtained the new ultrathin bilayer-coated micro-carriers for controlled oral delivery of 5-aminosalicylic acid from the safe, biodegradable, and easily available polysaccharides. 5-ASA was efficiently encapsulated in the synthesized microspheres. The particles are spherical with smooth surface and sizes in the range of several micrometers, as confirmed by optical microscopy and SEM measurements. The effect of the chitosan layer and bilayer coating on the release profiles of 5-ASA was studied at $\mathrm{pH}$ characteristic for stomach and intestine. Both the thin layers influence the release profile significantly. Chitosan/carrageenan coating slows down the release of 5-ASA considerably at both $\mathrm{pH}$. The release profile suggests that the process is a two-stage phenomenon. After the first $2 \mathrm{~h}$ (fast delivery), the slow sustained release is observed up to $8 \mathrm{~h}$. 5-ASA-containing alginate micro-carriers gained the additional protection against acidic conditions of stomach and the drug release in the intestine can be better controlled. The Weibull model was fitted to all experimentally obtained profiles and the possible mechanism of 5-ASA release kinetics was discussed.

Acknowledgments Project operated within the Foundation for Polish Science Team Programme cofinanced by the EU European Regional Development Fund, PolyMed, TEAM/2008-2/6. 
Open Access This article is distributed under the terms of the Creative Commons Attribution Noncommercial License which permits any noncommercial use, distribution, and reproduction in any medium, provided the original author(s) and source are credited.

\section{References}

1. ChN Bernstein, Eaden J, Steinhart AH, Munkholm P, Gordon PH (2002) Cancer prevention in inflammatory bowel disease and the chemoprophylactic potential of 5-aminosalicylic acid. Inflamm Bowel Dis 8:356-361. doi:10.1097/00054725-200209000-00007

2. Fina D, Franchi L, Caruso R, Peluso I, Naccari GC, Bellinvia S, Testi R, Pallone F, Monteleone G (2006) 5-Aminosalicylic acid enhances anchorage-independent colorectal cancer cell death. Eur J Cancer 42:2609-2616. doi:10.1016/j.ejca.2006.03.030

3. Rousseaux C, Lefebvre B, Dubuquoy L, Lefebvre P, Romano O, Auwerx J (2005) Intestinal antiinflammatory effect of 5-aminosalicylic acid is dependent on peroxisome proliferator-activated receptor- $\gamma$. J Exp Med 201:1205-1215. doi:10.1084/jem.20041948

4. Stolfi C, Fina D, Caruso R, Caprioli F, Sarra M, Fantini MC, Rizzo A, Pallone F, Monteleone G (2008) Cyclooxygenase-2-dependent and -independent inhibition of proliferation of colon cancer cells by 5-aminosalicylic acid. Biochem Pharmacol 75:668-676. doi:10.1016/j.bcp.2007.09.020

5. Loftus EV, Kane SV, Bjorkman D (2004) Systematic review: short-term adverse effects of 5-aminosalicylic acid agents in the treatment of ulcerative colitis. Aliment Pharmacol Ther 19: 179-189. doi:10.1111/j.0269-2813.2004.01827

6. Lakatos LP, Lakatos L (2008) Once daily 5-aminosalicylic acid for the treatment of ulcerative colitis; are we there yet? Pharmacol Res 58:190-195. doi:10.1016/j.phrs.2008.08.003

7. George M, Abraham TE (2006) Polyionic hydrocolloids for the intestinal delivery of protein drugs: alginate and chitosan-a review. J Control Rel 114:1-14. doi:10.1016/j.jconrel.2006.04.017

8. Anal AK, Stevens WF (2005) Chitosan-alginate multilayer beads for controlled release of ampicillin. Int J Pharm 290:45-54. doi:10.1016/j.ijpharm.2004.11.015

9. Lin SY, Ayres JW (1992) Calcium alginate beads as core carriers of 5-aminosalicylic acid. Pharm Res 9:1128-1131. doi:10.1023/A:1015887318767

10. Ch Wang, Ye S, Dai L, Liu X, Tang Z (2007) Enzymatic desorption of layer-by-layer assembled multilayer films and effects on the release of encapsulated indomethacin microcrystals. Carbohydr Res 342:2237-2243. doi:10.1016/j.carres.2007.06.025

11. Caruso F (2001) Nanoengineering of particle surfaces. Adv Mater 13:11-22. doi:10.1002/15214095(200101)13:1<11:AID-ADMA11>3.0.CO;2-N

12. Higuchi T (1961) Rate of release of medicaments from ointment bases containing drugs in suspensions. J Pharm Sci 50:874-875. doi:10.1002/jps.2600501018

13. Ritger PL, Peppas NA (1987) A simple equation for description of solute release. I. Fickian and nonFickian release from non-swellable devices in the form of slabs, spheres, cylinders or discs. J Control Rel 5:23-26. doi:10.1016/0168-3659(87)90034-4

14. Papadopoulou V, Kosmidis K, Vachou M, Macheras P (2006) On the use of the Weibull function for the discernment of drug release mechanisms. Int J Pharm 309:44-50. doi:10.1016/j.ijpharm.2005.10.044

15. Kosmidis K, Argyrakis P, Macheras P (2003) Fractal kinetics in drug release from finite fractal matrices. J Chem Phys 119:6373-6377. doi:10.1063/1.160373 